Recepción: 15 / 04 / 2017

Ciencias Medio Ambientales

Aceptación: 01 / 05 / 2017

Artículo Científico

Publicación: 15 / 05 / 2017

\title{
Gestión de los residuos sólidos en Puerto El Morro
}

\author{
Management of solid waste in Puerto El Morro
}

\section{Gestão de resíduos sólidos em Puerto El Morro}

Ivonne N. Casilla-Salazar ivonne.casillas@ug.edu.ec

Segundo E. Delgado-Menoscal segundo_delgado_m@yahoo.com

Alexandra K. Rubira-Carvache ${ }^{\text {m }}$

katiuska.rubirac@ug.edu.ec

Rubén D. Ruiz-Andaluz ${ }^{\text {IV }}$ ruben.ruiza@ug.edu.ec

Correspondencia: ivonne.casillas@ug.edu.ec

Magister en Administración Ambiental; Ingeniera Comercial; Universidad de Guayaquil, Ecuador.

Magister en Administración Ambiental; Ingeniera Civil; Universidad de Guayaquil, Ecuador.

Magister en Ciencias con Énfasis en Manejo Sustentable de Recursos Bioacuaticos y el Medio Ambiente, Biólogo; Universidad de Guayaquil, Ecuador.

Iv. Economista, Universidad de Guayaquil, Ecuador. 


\section{Resumen}

El presente trabajo de investigación, se realizó en la zona costera de la comuna de Puerto El Morro, perteneciente al cantón de Guayaquil en la Provincia del Guayas. El punto principal de la investigación fue la evaluación ambiental en dicho zona, la cual se identificó en base a una inspección en campo y mediante la evaluación de las evidencias objetivas de todo el proceso, dando mayor importancia a la capacidad de carga turística y cumplimiento de la gestión ambiental del área de influencia.

Durante el desarrollo del presente trabajo de investigación se pudo identificar líneas de acción viables para la correcta disposición de los residuos sólidos generados en dicha zona y se planteó el reciclaje como alternativa de mejora ambiental y desarrollo económico de sus habitantes. La propuesta de un Programa para la prevención, reciclaje, reutilización de los residuos sólidos, se basa en la implementación de medidas de prevención y mitigación, contingencia, capacitaciones, relaciones comunitarias, entre otros planes, que servirán de base para el desarrollo turístico, ambiental y económico del recinto Puerto El Morro.

Palabras clave: Disposición final; gestión ambiental; medidas ambientales; reciclaje; residuos sólidos. 


\section{Abstract}

The present research was carried out in the coastal area of t Puerto El Morro (Guayas: Guayaquil). The main point of the research was performed an environmental assessment in this area, which it was identified based on a field inspection and by assessing the objective evidence of the whole process, giving greater importance to tourism carrying capacity and compliance with environmental management of the area of influence.

During the development of this research were able to identify viable actions for the proper disposal of solid wastes generated in that area and recycling was raised as an alternative for environmental improvement and economic development of its people. The propose of a Programmer for the prevention, recycling, reuse of solid wastes, is based on the implementation of prevention and mitigation, contingency training, community relations, among other plans, which serve as the basis for tourism, environmental development and economic development of the municipality of Puerto El Morro.

Key words: Disposal; environmental management; environmental measures; recycling; solid waste. 


\section{Resumo}

A presente pesquisa foi realizada na região costeira de Puerto El Morro (Guayas: Guayaquil). O ponto principal da pesquisa foi realizar uma avaliação ambiental nesta área, que foi identificada com base em uma inspeção de campo e avaliando a evidência objetiva de todo o processo, dando maior importância à capacidade de transporte turístico e ao cumprimento da gestão ambiental da área De influência.

Durante o desenvolvimento desta pesquisa foram capazes de identificar ações viáveis para o descarte adequado de resíduos sólidos gerados nessa área ea reciclagem foi levantada como uma alternativa para melhoria ambiental e desenvolvimento econômico de sua população. A proposta de um Programa de prevenção, reciclagem e reutilização de resíduos sólidos baseia-se na implementação de ações de prevenção e mitigação, treinamento de contingência, relações comunitárias, entre outros planos, que servem de base para o turismo, desenvolvimento ambiental e desenvolvimento econômico de O município de Puerto El Morro.

Palavras chave: Disposição; gestão ambiental; medidas ambientais; reciclando; lixo sólido. 


\section{Introducción.}

La comuna rural de Puerto El Morro está ubicada en la provincia del Guayas, a 106 Km. De Guayaquil y a $5 \mathrm{Km}$. de la cabecera parroquial el Morro, su principal actividad es la pesca, posee una exuberante vegetación y está rodeada de manglares e islas. Al tratarse de una zona con mucha afluencia de turistas locales y extranjeros, no se cuenta con un programa de clasificación y control de los residuos sólidos urbanos, por ello se planteó la ejecución del presente proyecto en esta comuna perteneciente al cantón Guayaquil. (Cruz Sotelo \& Ojeda Benítez, 2013)

Los ingresos económicos de los pobladores de Puerto El Morro proceden principalmente de la pesca artesanal, turismo, reparación de embarcaciones, comercio local, agricultura de baja escala y otras actividades relacionadas con las parroquias cercanas. (Buenrostro Delgado \& Israde, 2003)

Para determinar la localización y las condiciones ambientales actuales de la comuna de Puerto El Morro, se procedió a realizar una inspección in situ y hacer el levantamiento de la información en campo, realizando entrevista a los actores principales, evidenciando una inadecuada disposición de los residuos sólidos, estas actividades se realizaron en un lapso de cinco días. Para validar el cumplimiento ambiental en cuanto a la disposición de residuos sólidos en esta zona costera, se identificaron los hallazgos encontrados en la Normativa Ambiental. (Jiménez Martínez, 2015)

En este lugar no se evidenció una adecuada disposición de los residuos sólidos, de tal manera que en este trabajo de investigación se dio a conocer las alternativas de gestión ambiental para el buen uso y adecuado manejo de los residuos sólidos generados por los turistas locales y extranjeros que visitan este lugar para conectarse con la naturaleza o disfrutar simplemente de su belleza paisajística. 
El presente estudio pretendió determinar adecuadas líneas de acción para la prevención de la contaminación ambiental por residuos sólidos y su correcta disposición final o aprovechamiento sostenible para los comuneros de la zona costera de la comuna Puerto El Morro.

\section{Materiales y métodos.}

\section{Diseño de la investigación}

La investigación comprendió un lapso de 6 meses, la misma que se efectuó en el período comprendido entre Octubre 2014 a Marzo 2015. Desde el punto de vista científico la investigación es un método puramente sistemático y metódico dirigido peculiarmente a la solución de un problema, la investigación puede categorizarse en tres tipos la investigación exploratoria, la descriptiva y la explicativa, con el fin de designar el tipo de investigación a utilizar en la investigación, se deberá analizar los tipos de estudio exploratorios y descriptivos. (Muñoz, 2013):

Exploratorios.- Se utilizan cuando existen temas que requieren de un levantamiento de información en relación a temas no estudiados o en vista de que no existe el suficiente conocimiento sobre el mismo, bajo lo cual se sentaran las bases para futuros estudios.

Bajo la siguiente consigna el tipo de investigación para determinar el resultado requerido y/o deseado en base al tema de investigación es de carácter explicativo, pues se establece como fin la medición del grado de relación entre las variables expuestas en la hipótesis. Según lo define (Talaya \& Molina, 2014) 


\section{Tipo de Investigación}

En relación al previo análisis de los resultados y al desarrollo de la investigación, se procedió a identificar los siguientes tipos de diseño de investigación: (Hurtado \& Toro, 2009):

a) No experimental: En este tipo de estudio no se genera la manipulación de las variables, entendiéndose que el investigador no puede ejercer ninguna influencia sobre las mismas para la obtención de información.

b) Cuasi-experimental: No tiene tan alto grado de control de las variables como el diseño experimental. Es decir que existe un cierto grado de influencia sobre las variables por parte del investigador.

c) Experimental: Establece una relación de causa y efecto en base a un fenómeno u objeto de estudio por medio de procedimientos que manipulan y controlan las variables que influencia e inciden sobre el fenómeno en cuestión. Estableciendo que el investigador es capaz de controlar el entorno.

Se estableció que para el tema de investigación en cuestión, no se realizará ningún tipo de manipulación a las variables, por lo tanto el tipo de diseño de investigación seleccionado es la de tipo no experimental.

\section{Población y Muestra}

Para conocimiento se tiene en vista el concepto de (Icart, Fuentelsaz, \& Pulpón, 2009) que dice lo siguiente:

Es la población accesible, que se puede estudiar. Es un subconjunto de la población que está definida por unos criterios de selección que son los criterios de inclusión y exclusión. La selección 
de esta población ha de posibilitar el cumplimiento de los objetivos del estudio y ha de permitir generalizar los resultados obtenidos.

En la actualidad en la comuna Puerto El Morro, habitan 1802 personas (Instituto Nacional de Estadisticas y Censos, 2010), para lo cual se realizó el cálculo adecuado para tomar el número de muestra en base a la población total.

Instrumentos de la Investigación

Para el desarrollo de la investigación se utilizó como técnica de investigación las encuestas como (Alvira, 2011) que menciona que "La encuesta es esencialmente una técnica de recogida de información con una filosofía subyacente (lo que convierte en un método), pero admite muy diferentes diseños de investigación”.

En las encuestas se utilizó preguntas cerradas siguiendo la escala de Likert, que permitió la mejor tabulación de los datos.

También se utilizó la guía de observación.

\section{Enfoque de la investigación}

La investigación estuvo direccionada a los habitantes de la comuna Puerto El Morro, dado que ellos conocen la realidad de su comuna y son quienes generan los residuos en la zona, y manejo de los mismos; por lo tanto, mediante sus respuestas, se identificó las falencias del sistema de recolección, transporte y disposición final de los residuos generados en el área de influencia.

\section{Criterios para la elaboración de la propuesta}


Ivonne N. Casilla-Salazar; Segundo E. Delgado-Menoscal; Alexandra K. Rubira-Carvache; Rubén D. Ruiz-Andaluz

Una vez efectuado el análisis y la interpretación de los resultados arrojados por las encuestas realizadas, se recopiló la información, que permitió detectar las falencias en la disposición de los residuos sólidos urbanos, y dio paso a la elaboración de un Programa para la prevención de la contaminación (reciclaje, reutilización y disposición final), que permita identificar los hallazgos encontrados y proponer la implementación de los diferentes planes que contiene el programa antes mencionado, para mejorar la calidad ambiental y desarrollo social de los habitantes de Puerto El Morro.

\section{Resultados.}

Siguiendo la metodología de investigación, se procedió con la aplicación de las encuestas a 317 personas de la comuna Puerto El Morro, de acuerdo a la metodología aplicada para la obtención de la muestra sobre la población total, y contar con un margen de error de un $+/$ - del $5 \%$.

Con el propósito de determinar las condiciones actuales de recolección y disposición final de los residuos sólidos urbanos, y posterior repercusión en la zona costera, medidas de prevención ambiental.

Para realizar un análisis adecuado se realizó una encuesta que abarcó desde lo general hasta lo especifico, como gestión, manejo y disposición de los residuos, como parte del proyecto (GESTIÓN DE RESIDUOS SÓlIDOS URBANOS, EFECTO DE LOS RESIDUOS SÓLIDOS, COMPOSICIÓN DE R.S.U. / GESTIÓN SUSTENTABLE), para poder tener una idea más clara de que línea a seguir según el impacto causado por los desechos y la percepción de la comunidad sobre esta problemática analizada.

Resultados de las encuestas realizadas a los habitantes de la comuna Puerto El Morro 
A continuación se detallan con tablas y gráficos resumidos de las encuestas realizadas a los habitantes de la comuna Puerto El Morro sobre la problemática ambiental en su área de influencia y contar con información de una fuente primaria, para lo cual las respuestas sistematizadas son las siguientes:

Pregunta 1: ¿A través de que medio usted se desprende de los R.S.U.?

\begin{tabular}{|c|c|c|}
\hline Opción de respuesta & No. de encuestados & \% de encuestados \\
\hline Carro recolector & 257 & $81 \%$ \\
\hline Incinerándolos & 27 & $9 \%$ \\
\hline Arrojan al río o canal & 0 & $0 \%$ \\
\hline Arrojan a terrenos baldíos & 26 & $8 \%$ \\
\hline Otros & 7 & $2 \%$ \\
\hline Total & $\mathbf{3 1 7}$ & $\mathbf{1 0 0 \%}$ \\
\hline
\end{tabular}

Cuadro $N^{\circ}$ 1.- Tipos de desprendimiento de los R.S.U.

La mayor parte de la población en Puerto El Morro entrega la basura al carro recolector, sin embargo al realizar las encuestas se pudo identificar que aún existen algunas personas que incineran, arrojan a terrenos baldíos o se desprenden de otra manera de los residuos sólidos que generan en su hogar.

Pregunta 2: ¿Usted se desprende de los Residuos Sólidos Urbanos por un medio diferente al planteado por el Gobierno Autónomo Descentralizado Municipal de Guayaquil?

\begin{tabular}{|c|c|c|}
\hline Opción de respuesta & No. de encuestados & \% de encuestados \\
\hline $\mathrm{Si}$ & 64 & $20 \%$ \\
\hline $\mathrm{No}$ & 253 & $80 \%$ \\
\hline Total & $\mathbf{3 1 7}$ & $\mathbf{1 0 0 \%}$ \\
\hline
\end{tabular}

Cuadro $N^{\circ}$ 2.- Desprendimiento de desechos diferentes a los Municipales 
La mayoría de los habitantes hace uso del servicio municipal de recolección de basura, sin embrago, como se indicó en el análisis anterior existen personas que realizan otro tipo métodos que contaminan el aire y suelo de la comunidad, aparte de irrespetar las ordenanzas municipales del cantón Guayaquil.

Pregunta 3: ¿Existen en su zona recicladores o chamberos que hurguen en sus residuos después que usted los ha sacado?

\begin{tabular}{|c|c|c|}
\hline Opción de respuesta & No. de encuestados & \% de encuestados \\
\hline $\mathrm{Si}$ & 174 & $55 \%$ \\
\hline $\mathrm{No}$ & 143 & $45 \%$ \\
\hline Total & $\mathbf{3 1 7}$ & $\mathbf{1 0 0 \%}$ \\
\hline
\end{tabular}

Cuadro $N^{\circ}$ 3.- Presencia de recicladores

En Puerto El Morro no hay presencia de recicladores o chamberos establecidos, más bien, las personas en su casa realizan la separación de plásticos y al momento de llegar camionetas provenientes del cantón Playas voceando la compra de plástico, estos la venden.

\section{Pregunta 4: ¿Existen en su zona alcantarillas que se tapen por la mala deposición de R.S.U.?}

\begin{tabular}{|c|c|c|}
\hline Opción de respuesta & No. de encuestados & \% de encuestados \\
\hline $\mathrm{Si}$ & 164 & $52 \%$ \\
\hline No & 153 & $48 \%$ \\
\hline Total & $\mathbf{3 1 7}$ & $\mathbf{1 0 0 \%}$ \\
\hline
\end{tabular}

Cuadro $N^{\circ}$ 4.- Alcantarillas tapadas por basura

En Puerto El Morro solo hay una alcantarilla que está ubicada en la calle principal, según las inspecciones realizadas, no se ha concluido el trabajo, debido a que existe presencia de tierra y no están asfaltadas las calles adyacentes a la avenida principal de acceso a la zona de estudio. 
Pregunta 5: ¿Merodean perros, gatos, e incluso roedores cerca de los R.S.U.?

\begin{tabular}{|c|c|c|}
\hline Opción de respuesta & No. de encuestados & \% de encuestados \\
\hline $\mathrm{Si}$ & 235 & $74 \%$ \\
\hline $\mathrm{No}$ & 82 & $26 \%$ \\
\hline Total & $\mathbf{3 1 7}$ & $\mathbf{1 0 0 \%}$ \\
\hline
\end{tabular}

Cuadro $N^{\circ}$ 5.- Presencia de animales que dispersen la basura

En el lugar merodean perros y gatos cuando los habitantes sacan su basura antes de que pase el carro recolector, y estos aspergean la basura en búsqueda de alimentos.

Una problemática adicional que se puedo identificar es la masiva presencia de cerdos en las calles de Puerto El Morro.

Pregunta 6: ¿Hay presencia de malos olores a causa de los R.S.U. en los alrededores?

\begin{tabular}{|c|c|c|}
\hline Opción de respuesta & No. de encuestados & \% de encuestados \\
\hline $\mathrm{Si}$ & 219 & $69 \%$ \\
\hline $\mathrm{No}$ & 98 & $31 \%$ \\
\hline Total & $\mathbf{3 1 7}$ & $\mathbf{1 0 0 \%}$ \\
\hline
\end{tabular}

Caudro $N^{\circ}$ 6.- Presencia de malos olores por basura

La presencia de los malos olores se da en mayor ocurrencia cuando pasa el carro recolector de basura, se debe al lixiviado que se produce por la descomposición de la materia orgánica y el calor del medio día.

Pregunta 7: ¿Existen enfermedades a causa de una gestión inadecuada de los R.S.U.?

\begin{tabular}{|c|c|c|}
\hline Opción de respuesta & No. de encuestados & \% de encuestados \\
\hline $\mathrm{Si}$ & 105 & $33 \%$ \\
\hline No & 212 & $67 \%$ \\
\hline Total & $\mathbf{3 1 7}$ & $\mathbf{1 0 0 \%}$ \\
\hline
\end{tabular}

Cuadro $N^{\circ}$ 7.- Enfermedades presentadas por basura 
Según los encuestados no hay mayor presencia de enfermedades causadas por la mala disposición de los R.S.U., sin embargo, manifestaron que se producían enfermedades a la dermis por el agua.

\section{Pregunta 8: ¿En su hogar se realiza una separación selectiva de Materia orgánica?}

\begin{tabular}{|c|c|c|}
\hline Opción de respuesta & No. de encuestados & \% de encuestados \\
\hline $\mathrm{Si}$ & 67 & $21 \%$ \\
\hline $\mathrm{No}$ & 250 & $79 \%$ \\
\hline Total & $\mathbf{3 1 7}$ & $\mathbf{1 0 0 \%}$ \\
\hline
\end{tabular}

\section{Cuadro $N^{\circ}$ 8.- Separación selectiva de materia orgánica}

La mayor parte de la población no realiza la separación selectiva de la materia orgánica, pero una parte de ellos, realiza la separación de estos desechos para darle de comer la denominada "labaza" a los cerdos.

Pregunta 9: ¿En su hogar se realiza una separación selectiva de plásticos?

\begin{tabular}{|c|c|c|}
\hline Opción de respuesta & No. de encuestados & \% de encuestados \\
\hline $\mathrm{Si}$ & 121 & $38 \%$ \\
\hline $\mathrm{No}$ & 196 & $62 \%$ \\
\hline Total & $\mathbf{3 1 7}$ & $\mathbf{1 0 0 \%}$ \\
\hline
\end{tabular}

Caudro $N^{\circ}$ 9.- Separación selectiva de plásticos

La mayoría deposita los plásticos en un bote común de desechos, pero existen personas que recopilan los plásticos pet en un saco, para venderlos posteriormente a los recicladores que provienen del cantón Playas. 
Pregunta 10: ¿En su hogar se realiza una separación selectiva de papel y/o cartón?

\begin{tabular}{|c|c|c|}
\hline Opción de respuesta & No. de encuestados & \% de encuestados \\
\hline $\mathrm{Si}$ & 62 & $20 \%$ \\
\hline No & 255 & $80 \%$ \\
\hline Total & $\mathbf{3 1 7}$ & $\mathbf{1 0 0 \%}$ \\
\hline
\end{tabular}

Cuadro $N^{\circ}$ 10.- Separación selectiva de papel y/o cartón

La mayoría deposita el papel y/o cartón en un bote común de desechos, pero hay una pequeña parte población que lo recopila para venderlos a los recicladores que provienen del cantón Playas.

Pregunta 11: ¿En su hogar se realiza una separación selectiva de metales u otros residuos?

\begin{tabular}{|c|c|c|}
\hline Opción de respuesta & No. de encuestados & \% de encuestados \\
\hline $\mathrm{Si}$ & 49 & $15 \%$ \\
\hline No & 268 & $85 \%$ \\
\hline Total & $\mathbf{3 1 7}$ & $\mathbf{1 0 0 \%}$ \\
\hline
\end{tabular}

Cuadro $N^{\circ}$ 11.- Separación selectiva de metales $u$ otros residuos

Pocas personas dijeron que separaban los metales o los envases enlatados, y la mayoría manifestó que preferían echarlos en un bote en común con los demás desechos.

Pregunta 12: ¿Qué hace con los residuos sólidos separados en la fuente?

\begin{tabular}{|c|c|c|}
\hline Opción de respuesta & No. de encuestados & \% de encuestados \\
\hline Vende a recicladores & 46 & $15 \%$ \\
\hline Regala a quién lo necesita & 110 & $35 \%$ \\
\hline Echa a la basura & 161 & $51 \%$ \\
\hline Total & $\mathbf{3 1 7}$ & $\mathbf{1 0 0 \%}$ \\
\hline
\end{tabular}

Cuadro $N^{\circ}$ 12.- Destino de los desechos separados 
La mayor parte de la población indicó que echa a la basura los desechos separados o simplemente no realiza la separación en la fuente y coloca todo en un tacho en común; otra parte manifestó que lo que separaba lo regalaba a vecinos, refiriéndose a la materia orgánica y plásticos; en menor proporción, dijeron que lo vendían a los recicladores.

Pregunta 13: ¿Qué cantidad de materia orgánica se genera en su hogar semanalmente?

\begin{tabular}{|c|c|c|}
\hline Opción de respuesta & No. de encuestados & \% de encuestados \\
\hline $0-1 \mathrm{Kg}$ & 35 & $11 \%$ \\
\hline $2-3 \mathrm{Kg}$ & 137 & $43 \%$ \\
\hline $4-5 \mathrm{Kg}$ & 133 & $42 \%$ \\
\hline Más de $5 \mathrm{Kg}$ & 12 & $4 \%$ \\
\hline Total & $\mathbf{3 1 7}$ & $\mathbf{1 0 0 \%}$ \\
\hline
\end{tabular}

\section{Cuadro $N^{\circ}$ 13.- Generación de materia orgánica semanalmente}

El promedio de generación de materia orgánica en Puerto El Morro, está en 4kg por cada semana.

Pregunta 14: ¿Qué cantidad de plásticos se generan en su hogar semanalmente?

\begin{tabular}{|c|c|c|}
\hline Opción de respuesta & No. de encuestados & \% de encuestados \\
\hline $0-1 \mathrm{Kg}$ & 199 & $63 \%$ \\
\hline $2-3 \mathrm{Kg}$ & 107 & $34 \%$ \\
\hline $4-5 \mathrm{Kg}$ & 11 & $3 \%$ \\
\hline Más de $5 \mathrm{Kg}$ & 0 & $0 \%$ \\
\hline Total & $\mathbf{3 1 7}$ & $\mathbf{1 0 0 \%}$ \\
\hline
\end{tabular}

\section{Cuadro $N^{\circ}$ 15.- Generación de plásticos semanalmente}

El promedio de generación de desechos plásticos es de $1.5 \mathrm{Kg}$ por cada semana, según lo indicado por los encuestados. 
Pregunta 15: ¿Qué cantidad de papel y/o cartón se generan en su hogar semanalmente?

\begin{tabular}{|c|c|c|}
\hline Opción de respuesta & No. de encuestados & \% de encuestados \\
\hline $0-1 \mathrm{Kg}$ & 247 & $78 \%$ \\
\hline $2-3 \mathrm{Kg}$ & 48 & $15 \%$ \\
\hline $4-5 \mathrm{Kg}$ & 17 & $5 \%$ \\
\hline Más de $5 \mathrm{Kg}$ & 5 & $2 \%$ \\
\hline Total & $\mathbf{3 1 7}$ & $\mathbf{1 0 0 \%}$ \\
\hline
\end{tabular}

Cuadro $N^{\circ}$ 15.- Generación de papel y/o cartón semanalmente

La mayoría de los encuestados dijeron que producían menos de $1 \mathrm{~kg}$ de papel y/o cartón por cada semana, salvo las personas que tenían negocios o estaban realizando alguna actividad de subsistencia en específico.

Pregunta 16: ¿Qué cantidad de metales se generan en su hogar semanalmente?

\begin{tabular}{|c|c|c|}
\hline Opción de respuesta & No. de encuestados & \% de encuestados \\
\hline $0-1 \mathrm{Kg}$ & 292 & $92 \%$ \\
\hline $2-3 \mathrm{Kg}$ & 25 & $8 \%$ \\
\hline $4-5 \mathrm{Kg}$ & 0 & $0 \%$ \\
\hline Más de $5 \mathrm{Kg}$ & 0 & $0 \%$ \\
\hline Total & $\mathbf{3 1 7}$ & $\mathbf{1 0 0 \%}$ \\
\hline
\end{tabular}

Cuadro $N^{\circ}$ 16.- Generación de metales o similares semanalmente

La población indicó que no generaban metales o envases enlatados porque en el lugar prefieren servirse los alimentos frescos. 
Pregunta 17: ¿Qué otro tipo de residuos se generan en su hogar semanalmente?

\begin{tabular}{|c|c|c|}
\hline Opción de respuesta & No. de encuestados & \% de encuestados \\
\hline Pañales & 84 & $26 \%$ \\
\hline Maderas & 15 & $5 \%$ \\
\hline Llantas & 17 & $5 \%$ \\
\hline Vidrio & 97 & $31 \%$ \\
\hline Material de construcción & 6 & $2 \%$ \\
\hline Otros & 98 & $31 \%$ \\
\hline Total & $\mathbf{3 1 7}$ & $\mathbf{1 0 0 \%}$ \\
\hline
\end{tabular}

Cuadro $N^{\circ}$ 17.- Generación de otro tipo de residuos semanalmente

La mayor parte de la población señaló que generaban mayor cantidad de vidrio por las bebidas embotelladas que compran en tiendas aledañas, seguido por pañales al haber incidencia de maternidad a edad precoz, además indicaron en "otros" desechos como cauchos, fundas de supermercado, vasos y platos plásticos, cajas de tabaco, etc.

Pregunta 18: ¿Recibe usted capacitaciones de educación ambiental, sobre la separación en la fuente de R.S.U.?

\begin{tabular}{|c|c|c|}
\hline Opción de respuesta & No. de encuestados & \% de encuestados \\
\hline $\mathrm{Si}$ & 50 & $16 \%$ \\
\hline $\mathrm{No}$ & 267 & $84 \%$ \\
\hline Total & $\mathbf{3 1 7}$ & $\mathbf{1 0 0 \%}$ \\
\hline
\end{tabular}

Cuadro $N^{\circ}$ 18.- Asistencia a capacitación ambiental

Una pequeña parte de la población se ha beneficiado de capacitaciones ambientales impartidas por diferentes organismos privados o gubernamentales; por otro lado, la gran mayoría manifestó que no han recibido capacitaciones especializadas en ambiente e indicaron que les gustaría participar en este tipo de capacitaciones o talleres. 
Pregunta 19: ¿Considera usted, que una mala disposición de los R.S.U. produce un impacto negativo al medio ambiente?

\begin{tabular}{|c|c|c|}
\hline Opción de respuesta & No. de encuestados & \% de encuestados \\
\hline $\mathrm{Si}$ & 317 & $100 \%$ \\
\hline $\mathrm{No}$ & 0 & $0 \%$ \\
\hline Total & $\mathbf{3 1 7}$ & $\mathbf{1 0 0 \%}$ \\
\hline
\end{tabular}

\section{Cuadro $N^{\circ}$ 20.- Percepción del impacto ambiental causado por la basura}

Todas las personas encuestadas coincidieron que una mala disposición de los residuos sólidos urbanos produce un impacto negativo al ambiente, porque afectan a la belleza paisajística, tapan la alcantarilla e incluso pueden llegar a los esteros y causar contaminación de los productos pesqueros como son los mariscos que se generan en la zona.

Pregunta 20: ¿Estaría usted de acuerdo en contribuir a un manejo sustentable de los R.S.U. para reducir los impactos al medio ambiente?

\begin{tabular}{|c|c|c|}
\hline Opción de respuesta & No. de encuestados & \% de encuestados \\
\hline $\mathrm{Si}$ & 317 & $100 \%$ \\
\hline No & 0 & $0 \%$ \\
\hline Total & $\mathbf{3 1 7}$ & $\mathbf{1 0 0 \%}$ \\
\hline
\end{tabular}

\section{Cuadro $N^{\circ}$ 20.- Predisposición a contribuir con el medio ambiente}

La mayoría de la población sondeada, indico que están de acuerdo en contribuir con el manejo sustentable de los residuos sólidos urbanos, para reducir los impactos que estos causan a su entorno, aclarando que se les debe dar las facilidades para poder realizarlo, porque son personas de escasos recursos. 
Es importante recalcar, que dentro de la encuesta no se consideró al vidrio, ya que conversaciones previas con algunos de los ciudadanos de Puerto El Morro indicaron a la investigación que no consumían en demasía bebidas o productos envasados en vidrio, por ello se lo consideró únicamente en la pregunta No.17: ¿Qué otro tipo de residuos se generan en su hogar semanalmente?, cuya sistematización indica que el 31\% de los ciudadanos encuestados generan este tipo de desecho.

\section{Conclusiones.}

A continuación se detallan las conclusiones resultantes del presente estudio realizado en la comuna Puerto El Morro:

Se identificó el área de influencia, evidenciando problemas de contaminación a los recursos naturales de Puerto el Morro, además de hacer el levantamiento de la línea base y recopilo la información primaria y secundaria relacionada con la problemática que dieron paso al presente estudio.

Se realizó una caracterización de los residuos sólidos en Puerto El Morro, a través de una minga de limpieza con los pobladores del lugar, evidenciando los siguientes desechos comunes: Orgánico, papel - cartón, plásticos (botellas, fundas, etc.), metal, vidrio, etc., esto como consecuencia de malos hábitos de los turistas en cuanto a la disposición de los desechos sólidos y la falta de sensibilización ambiental de los habitantes de dicha localidad.

Se establecieron varias líneas de acción para lograr una correcta disposición de los desechos sólidos en esta zona costera de Guayaquil, que permita prevenir y mitigar los impactos ambientales ya existentes. 
Se analizaron las alternativas más viables tanto en lo técnico y económico y en cumplimento de la normativa legal, para el logro del objetivo planteado, por lo que se elaboró una propuesta para prevención de la contaminación, la misma que se detalla más adelante.

En Puerto El Morro hay un sistema de recolección de basura, que lo realiza la concesionaria Puerto Limpio, pero presenta ciertas falencias como en el barrido, esta actividad se lo hace únicamente en la calle principal y no en las adyacentes, por lo que igual existe desechos sólidos esparcidos por las calles, se concluye que la M.I. Municipalidad de Guayaquil debe exigir a la concesionaria Puerto Limpio optimice este servicio y coadyuvar al cumplimiento de lo planteado en el Programa Nacional para la Gestión Integral de Desechos Sólidos - PNGIDS ECUADOR, que viene impulsando el Ministerio del Ambiente desde el mes de abril del año 2010.

Durante la investigación se puedo constatar que otro problema existente en el área de estudio es la falta de infraestructura y vías de acceso; a pesar de promoverse a Puerto El Morro como un atractivo turístico por su abundante biodiversidad por fauna y flora, no cuenta con todos los servicios básicos, a criterio de la investigación es fundamental que la autoridades pertinentes atiendan estas necesidades básicas insatisfechas para dotarles de una mejor calidad de vida a este segmento desatendido del cantón Guayaquil.

Además de lo indicado en el párrafo anterior, se evidenció demasiados cerdos sueltos, los cuales se los podía visualizar desde en ingreso a Puerto El Morro hasta la zona del muelle, para lo cual se debe concientizar a los habitantes sobre la importancia de tener su ganado porcino encerrado en un corral, para que no contamine el ambiente ni cause perjuicios dañando las bolsas o sacos de basura que sacan los pobladores antes de que pase el carro recolector de Puerto Limpio. 
Ivonne N. Casilla-Salazar; Segundo E. Delgado-Menoscal; Alexandra K. Rubira-Carvache; Rubén D. Ruiz-Andaluz

Según las conversaciones preliminares sostenidas con los habitantes, no existe una preocupación por parte de los GAD’s pertinentes para concienciarlos mediante charlas o capacitaciones en temas ambientales y específicamente sobre la clasificación en la fuente y disposición final de los residuos sólidos urbanos.

Los habitantes de Puerto El Morro no tienen una cultura de reciclaje en la fuente, y desconocen los beneficios económicos y ambientales que pueden obtener si seleccionarán los materiales valorizables y entregarlos directamente para el reciclaje. Se cree que con una correcta estrategia se disminuirá desde el origen la cantidad de residuos, repercutiendo en un ahorro de recursos destinados a la disposición final, al tiempo que se alcanzaría el objetivo planteado para nuestro caso particular, cual es mejorar la calidad de vida de los pobladores de la Provincia del Guayas.

\section{Criterios para la propuesta.}

Se consideró los siguientes puntos: educación a los habitantes, recolección de los R.S.U. , transporte de los R.S.U., tipos de residuos sólidos urbanos generados, modelo de gestión, relleno sanitario.

\section{Bibliografía.}

Alvira, F. (2011). La Encuesta: Una perspectiva Metodológica generales. Madrid: CIS.

Buenrostro Delgado, O., \& Israde, I. (2003). La gestión de los residuos sólidos municipales en la cuenca del lago de Cuitzeo, México. Revista Internacional de Contaminación Ambiental, 19(4), 161-169.

Cruz Sotelo, S., \& Ojeda Benítez, S. (2013). Gestión sostenible de los residuos sólidos urbanos. Revista Internacional de Contaminación Ambiental, 29(3).

Hurtado, I., \& Toro, J. (2009). Paradigmas y métodos de investigación en tiempos de cambio. Venezuela: CEC, S.A. 
Icart, M., Fuentelsaz, C., \& Pulpón, A. (2009). Elaboración y presentación de un proyecto de investigación. España: Ediciones Ub.

Instituto Nacional de Estadisticas y Censos. (Noviembre de 2010). VII Censo de Población y VI de Vivienda INEC. Ecuador.

Jiménez Martínez, N. (2015). La gestión integral de residuos sólidos urbanos en México: entre la intención y la realidad. Revista Latinoamericana de Estudios Socioambientales, 17(1), Revista Latinoamericana de Estudios Socioambientales.

Muñoz, R. (2013). Etapas del plan de marketing. Madrid: Centro de estudios financieros.

Talaya, A., \& Molina, A. (2014). Investigación de mercados. Madrid: ESIC Editorial. 